\title{
Effect of Tai Chi exercise on blood lipid profiles: a meta-analysis of randomized controlled trials
}

\author{
Xiao-hong $\mathrm{PAN}^{1}$, Amina MAHEMUTI ${ }^{2}$, Xue-hua ZHANG ${ }^{1}$, Ya-ping $\mathrm{WANG}^{1}$, Po $\mathrm{HU}^{1}$, \\ Ju-bo JIANG ${ }^{1}$, Mei-xiang XIANG ${ }^{1}$, Gang LIU $^{2}$, Jian-an WANG ${ }^{\dagger 1}$ \\ ( ${ }^{1}$ Department of Cardiology, the Second Affiliated Hospital, School of Medicine, Zhejiang University, Hangzhou 310009, China) \\ ('Department of Cardiology, the First People's Hospital of Aksu District in Xinjiang Uygur Autonomous Region, Aksu 843000, China) \\ †E-mail: wja@zju.edu.cn \\ Received Feb. 1, 2016; Revision accepted July 14, 2016; Crosschecked July 18, 2016
}

\begin{abstract}
Objective: Studies have demonstrated that Tai Chi exercise improves blood lipid level with inconsistent results. A meta-analysis was conducted to quantify the effects of Tai Chi on blood lipid profiles in humans. Methods: We screened the databases of PubMed, EMBASE, Cochrane Library (Central), Web of Science, China National Knowledge Infrastructure (CNKI), Wanfang data, and Clinicaltrials.gov for randomized controlled trials with Physiotherapy Evidence Database (PEDro) score more than 3 points up to June 2015. Six studies involving 445 subjects were included. Most trials applied 12-week Tai Chi intervention courses. Results: In comparison with the control group, blood triglyceride (TG) level difference between follow-up and baseline was statistically significantly lower in the Tai Chi practicing group (weighted mean difference (WMD) $-16.81 \mathrm{mg} / \mathrm{dl}$; $95 \%$ confidence intervals $(\mathrm{Cl})-31.27$ to $-2.35 \mathrm{mg} / \mathrm{dl} ; P=0.02)$. A trend to improving total cholesterol (TC) reduction was found with Tai Chi (WMD $-7.96 \mathrm{mg} / \mathrm{dl}$; $95 \% \mathrm{Cl}-17.30$ to $1.39 \mathrm{mg} / \mathrm{dl} ; P=0.10)$. However, no difference was found in blood low-density lipoprotein cholesterol (LDL-C) or high-density lipoprotein cholesterol (HDL-C). Conclusions: Tai Chi exercise lowered blood TG level with a trend to decrease blood TC level. Our data suggest that Tai Chi has the potential to implement meaningful blood lipid modification and serve as an adjunctive exercise modality. The relationship between Tai Chi exercise regimen and lipid profile change might have a scientific priority for future investigation.
\end{abstract}

Key words: Tai Chi, Blood lipid, Triglycerides, Meta-analysis http://dx.doi.org/10.1631/jzus.B1600052

CLC number: G852.11

\section{Introduction}

Dyslipidemia is a strong risk factor for coronary heart disease (CHD) and stroke, which was proven by previous clinical investigations (Mozaffarian et al., 2015). Elevated blood total cholesterol (TC), lowdensity lipoprotein cholesterol (LDL-C) or triglycerides (TG), and decreased blood high-density lipoprotein cholesterol (HDL-C) are associated with high incidence and worse prognosis (Reiner et al., 2011;

\footnotetext{
${ }^{\ddagger}$ Corresponding author

(iD) ORCID: Jian-an WANG, http://orcid.org/0000-0003-0409-8941

(c) Zhejiang University and Springer-Verlag Berlin Heidelberg 2016
}

Stone et al., 2014; Hu et al., 2015). Pharmaceutical therapy based on lifestyle modification plays an important role in dyslipidemia treatment, but accompanies with residual risks and potential adverse effects (Nicholls et al., 2014). Most positive observations from complementary and alternative medicine (CAM) therapies, such as Tai Chi exercise, were ever considered a placebo effect. However, growing evidences demonstrated the beneficial role of CAM in dyslipidemia and cardiovascular diseases, especially in those who participated in randomized clinical trials (Walach and Jonas, 2004; Frishman et al., 2009).

Tai Chi, originating from ancient China, is a form of physical exercise related to martial arts. It 
harmonizes Yin and Yang by slow and flowing movements with deep breathing and meditation (Frishman et al., 2009). Recently Tai Chi gained worldwide popularity as a consequence of multifaceted physical and psychological benefits, especially for the older population (Wayne and Kaptchuk, 2008). Studies have shown that Tai Chi improves lower extremity muscle strength and balance, prevents falls, and relieves pain (Hui et al., 2015). Tai Chi enhances aerobic capacity, immune function, and endothelial function (Frishman et al., 2009). Furthermore, Tai Chi exercise reduces CHD and stroke risks by benefiting the lowering of blood pressure and possibly lipid modification (Dalusung-Angosta, 2011).

The effects of Tai Chi on blood lipid in patients with dyslipidemia have been investigated in several randomized controlled trials (RCTs) (Tsai et al., 2003; Thomas et al., 2005; Lam et al., 2008; Zhang and Fu, 2008; Chen et al., 2010; Lu and Kuo, 2012). However, the sample sizes are relatively small, and results remain inconsistent and inconclusive. It is necessary to summarize current evidence regarding the effectiveness of Tai Chi on lipid levels in individuals. Our meta-analysis was conducted to address whether Tai Chi is an effective physical activity that improves blood lipid levels.

\section{Materials and methods}

We performed the meta-analysis according to the preferred reporting items for systematic reviews and meta-analyses (PRISMA) statement (Moher et al., 2009).

\subsection{Literature search}

The online databases PubMed, EMBASE, Cochrane Library (Central), Web of Science, China National Knowledge Infrastructure (CNKI), Wanfang data, and Clinicaltrials.gov were searched from their inception until June 2015 for relevant studies. If there are missing data or duplicated data in multiple publications to be clarified, authors were contacted.

\subsection{Search strategy}

A combination of the following terms was employed in the search strategy: (lipid or lipidemia or dyslipidemia or hyperlipidemia or cholesterol or hy- percholesterolemia or lipoprotein or dyslipoproteinemia or hyperlipoproteinemia or $L D L$ or low density lipoprotein or HDL or high density lipoprotein or triglyceride or hypertriglyceridemia) and (Tai Chi or taichi or tai-chi or tai ji or taiji or tai-ji or t'ai chi or taijiquan or taichiquan). The wile-card term "**" was applied. We imposed no language restrictions.

\subsection{Eligibility}

Original studies were included if the following criteria were met: (1) included adults 18 years or older; (2) were RCTs with ethical approval addressed; (3) investigated and reported the impact of any style of Tai Chi on blood concentrations of at least one of the lipid measures including TC, LDL-C, HDL-C, or TG; (4) had a duration of at least 4 weeks; and, (5) Physiotherapy Evidence Database (PEDro) score of the trial more than 3 points. Two reviewer authors (X.H. Zhang and Y.P. Wang) independently screened all the retrieved titles and abstracts, and then independently reviewed the full text of potentially relevant papers and conducted data abstraction. If disagreements persisted, a third review author (X.H. Pan) was consulted to reach consensus.

\subsection{Data extraction and synthesis}

The authors extracted data by a pre-piloted data form: first author, year of publication, study location, study purpose, participant characteristics, the details of Tai Chi (type, number of forms, duration, frequency, training length), circulating concentrations of blood lipids, and other outcome variables (baseline, post-intervention, and change values, if available).

Plasma lipid concentration values were collated in $\mathrm{mg} / \mathrm{dl}$. While expressed in $\mathrm{mmol} / \mathrm{L}$, the data of cholesterols (TC, LDL-C, HDL-C) and TG were multiplied by 38.6 and 88.5 , respectively (Reiner et al., 2011). Change in post-intervention mean was calculated by substracting the baseline from follow-up values. When data on standard deviation (SD) were not available after contacting the authors, imputation was used to approach it (Higgins and Deeks, 2011).

\subsection{Quality assessment}

Study quality was assessed using a modified PEDro scale (http://www.pedro.org.au/english/downloads/pedroscale) based on eligibility criteria, random allocation, concealed allocation, baseline similarity, blinding of 
participants, blinding of therapists, blinding of assessors, follow-up, intention-to-treat analysis, between-group statistical comparisons, and point measures and measures of variability (Maher et al., 2003). The evidence was hence categorized into three levels: low quality (0-3 points), moderate quality (4-7 points), and high quality (8-10 points). When the trial was deemed to be low quality, it was excluded (Verhagen et al., 1998).

Two reviewers (P. Hu and J.B. Jiang) independently assessed eligible studies for quality scores. Any discrepancies were discussed with a third reviewer (M.X. Xiang) until consensus was reached.

\subsection{Statistical analysis}

Review Manager software version 5.3 (the Nordic Cochrane Centre, the Cochrane Collaboration, Copenhagen, Denmark) and STATA 12.0 (Stata Corporation, College Station, TX, USA) were used for statistical analyses. Results were presented as weighted mean difference (WMD) with 95\% confidence interval (CI). Heterogeneity across studies was assessed by the Cochrane Chi-square test and $I^{2}$ statistic. It was categorized into no, low, moderate, and high heterogeneity with an $I^{2}$ statistic of $<25 \%$, $25 \%-50 \%, 50 \%-75 \%$, and $>75 \%$, respectively.
Significance value was set at Cochrane Chi-square $P<0.1$ or $I^{2}>50 \%$ (Higgins et al., 2003). A randomeffects model (REM) or a fixed-effects model (FEM) was applied when the heterogeneity was notable or not. Sensitivity analysis was implemented to assess the influence of individual trial by successively omitting each trial one at a time. Risk of publication bias was not evaluated due to limited study number $(<10)$ (Pan et al., 2013). A $P$-value $<0.05$ indicated statistically significant difference.

\section{Results}

\subsection{Study selection}

As shown in Fig. 1, of the 391 studies yielded by initial search, 86 were excluded at first inspection as duplicates, 103 were removed after reading titles, and 130 were removed after reading abstracts. Seventytwo studies were identified as potential inclusions. After full-text reviews, 22 trials were not randomized controlled design, and 38 trials were of low quality according to PEDro score. Three trials were excluded for Tai Chi combined with other interventions, no baseline data including lipid profiles were reported in
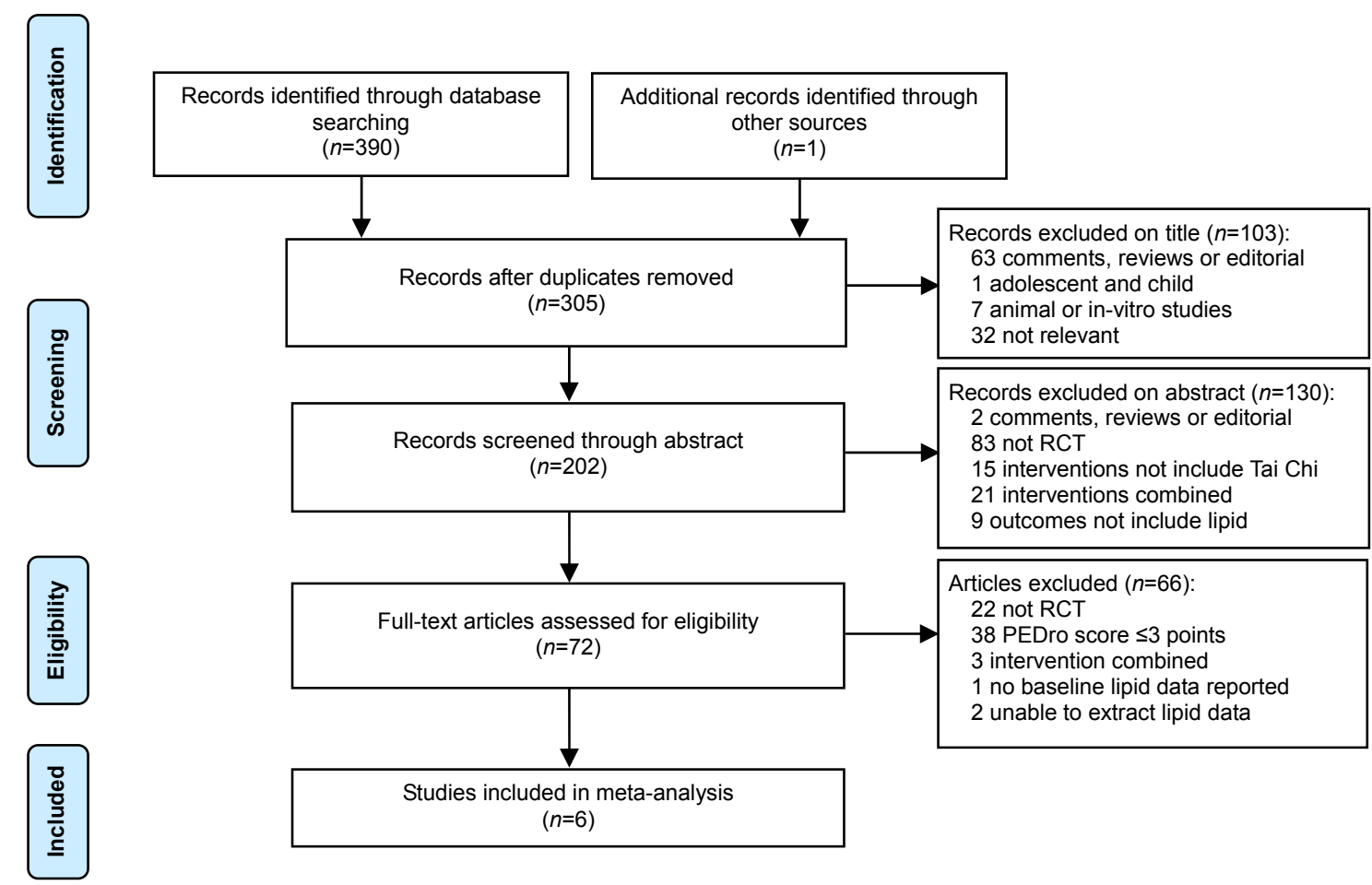

Fig. 1 Flow chart of the study selection process 
one trial, and it was not possible to extract lipid data from the outcomes in two trials. Six randomized placebo-controlled trials (Tsai et al., 2003; Thomas et al., 2005; Lam et al., 2008; Zhang and Fu, 2008; Chen et al., 2010; Lu and Kuo, 2012) fulfilled the inclusion criteria and were included in the final meta-analysis.

\subsection{Characteristics of included studies}

Six studies were included in the analysis representing a total sample of 445 participants. The included studies were all RCTs published in English, with one study being reported as blinded to assessors (Lam et al., 2008).

The characteristics of included studies are presented in Table 1. Most of the subjects were elderly Chinese. Subject characteristics included type 2 diabetes (Lam et al., 2008; Zhang and Fu, 2008; Chen et al., 2010) and obesity (Chen et al., 2010). The trial including obese subjects reported a significant reduction of body mass index (BMI) after practicing Tai Chi (Chen et al., 2010). No significant changes in BMI were reported in the remaining studies. Two studies reported that participants were advised to maintain their diet (Tsai et al., 2003; Chen et al., 2010). Regarding the type of Tai Chi, the Yang style was applied in 5 of the 6 studies (Tsai et al., 2003; Thomas et al., 2005; Lam et al., 2008; Zhang and Fu, 2008; $\mathrm{Lu}$ and Kuo, 2012), and one of them was mixed with Sun style (Lam et al., 2008). Chen style was introduced in one study (Chen et al., 2010). The forms of Tai Chi varied from 13 to 108 forms. The Tai Chi treatment programs ranged from 12 weeks to 12 months, and most were of 12 to 14 weeks' duration (Tsai et al., 2003; Zhang and Fu, 2008; Chen et al., 2010; Lu and Kuo, 2012). Session length ranged from 40 to $60 \mathrm{~min}$. Tai Chi training was usually practiced 3-5 times per week (Tsai et al., 2003; Thomas et al., 2005; Zhang and $\mathrm{Fu}, 2008$; Chen et al., 2010). However, participants were asked to attend Tai Chi training every day in the trial of $\mathrm{Lu}$ and Kuo (2012). Usual care (Tsai et al., 2003; Thomas et al., 2005; Zhang and $\mathrm{Fu}, 2008$; Lu and Kuo, 2012) or waitlist control (Lam et al., 2008) was the most common control group. Tai Chi exercise was compared to conventional exercise in the trial of Chen et al. (2010). All studies reported changes in lipid profiles including TC, LDL-C, HDL-C, and TG, except for the trials by Lam et al. (2008) and Chen et al. (2010). No studies reported major cardiovascular events. Good compliances were reported in all studies with no adverse events from Tai Chi practicing.

The majority of trials were of medium quality according to the PEDro scale, i.e., included articles had a PEDro score of 5 or 6 points, indicating moderate quality evidence.

\subsection{Effect of Tai Chi on TC}

All 6 trials reported data of TC change following the Tai Chi practicing program in 445 participants. Pooled blood TC level difference between follow-up and baseline decreased by WMD $-7.96 \mathrm{mg} / \mathrm{dl}$ in the Tai Chi group compared with the control group with no significant difference (REM: $95 \% \mathrm{CI},-17.30$ to

Table 1 Characteristics of included studies

\begin{tabular}{|c|c|c|c|c|c|c|c|c|c|c|}
\hline \multirow{2}{*}{ Study } & \multirow{2}{*}{ Participant } & \multirow{2}{*}{ Location } & \multirow{2}{*}{$\begin{array}{l}\text { No. } \\
\text { (Tai Chi/ } \\
\text { control) }\end{array}$} & \multirow{2}{*}{$\begin{array}{l}\text { Mean } \\
\text { age } \\
\text { (year) }\end{array}$} & \multirow{2}{*}{$\begin{array}{c}\text { Male } \\
(\%)\end{array}$} & \multicolumn{3}{|c|}{ Tai Chi group } & \multirow{2}{*}{ Follow-up } & \multirow{2}{*}{$\begin{array}{l}\text { PEDro } \\
\text { score } \\
\text { (points) }\end{array}$} \\
\hline & & & & & & Form or style & $\begin{array}{l}\text { Duration } \\
\text { (min) }\end{array}$ & Frequency & & \\
\hline $\begin{array}{l}\text { Tsai et al., } \\
\quad 2003\end{array}$ & $\begin{array}{l}\text { High-normal BP } \\
\text { or stage I HTN }\end{array}$ & Taiwan & $\begin{array}{c}76 \\
(37 / 39)\end{array}$ & 51.1 & 43.2 & $\begin{array}{l}108 \text { forms } \\
\text { Yang style }\end{array}$ & 50 & 3 times/week & 12 weeks & 5 \\
\hline $\begin{array}{l}\text { Thomas et al., } \\
2005\end{array}$ & Elderly & Hongkong & $\begin{array}{c}142 \\
(64 / 78)\end{array}$ & 69.0 & 54.9 & $\begin{array}{l}24 \text { forms } \\
\text { Yang style }\end{array}$ & 60 & 3 times/week & 12 months & 6 \\
\hline $\begin{array}{l}\text { Lam et al., } \\
\qquad 2008\end{array}$ & Type 2 diabetes & Sidney & $\begin{array}{c}53 \\
(28 / 25)\end{array}$ & 62.1 & 45.3 & $\begin{array}{l}20 \text { forms } \\
\text { Yang and } \\
\text { Sun styles }\end{array}$ & 60 & $\begin{array}{c}\text { Twice/week for } \\
3 \text { months+once/week } \\
\text { for } 3 \text { months }\end{array}$ & 6 months & 6 \\
\hline $\begin{array}{l}\text { Zhang and } \\
\text { Fu, } 2008\end{array}$ & $\begin{array}{l}\text { Type } 2 \text { diabetes } \\
\text { women }\end{array}$ & Beijing & $\begin{array}{c}20 \\
(10 / 10)\end{array}$ & 57.4 & 0 & $\begin{array}{l}24 \text { forms } \\
\text { Yang style }\end{array}$ & 60 & 5 times/week & 14 weeks & 5 \\
\hline $\begin{array}{l}\text { Chen et al., } \\
2010\end{array}$ & $\begin{array}{c}\text { Obese type } 2 \\
\text { diabetes }\end{array}$ & Taiwan & $\begin{array}{c}104 \\
(56 / 48)\end{array}$ & 58.5 & 43.3 & $\begin{array}{l}13 \text { forms } \\
\text { Chen style }\end{array}$ & 60 & 3 times/week & 12 weeks & 5 \\
\hline $\begin{array}{l}\text { Lu and Kuo, } \\
2012\end{array}$ & $\begin{array}{l}\text { Middle-aged and } \\
\text { elderly }\end{array}$ & Taiwan & $\begin{array}{c}50 \\
(25 / 25)\end{array}$ & 55.0 & 40.0 & $\begin{array}{l}64 \text { forms } \\
\text { Yang style }\end{array}$ & 40 & 7 times/week & 3 months & 5 \\
\hline
\end{tabular}

BP: blood pressure; HTN: hypertension 
$1.39 \mathrm{mg} / \mathrm{dl} ; P=0.10)$. Significant heterogeneity among the studies was detected $\left(I^{2}=81 \%, P<0.0001\right)$ (Fig. 2). Sensitivity analyses were subsequently performed to explore the origin of heterogeneity. Exclusion of the trial conducted by Thomas et al. (2005) changed the results, but did not resolve the heterogeneity (WMD $-11.09 \mathrm{mg} / \mathrm{dl} ; 95 \% \mathrm{CI}-19.68$ to $-2.50 \mathrm{mg} / \mathrm{dl} ; P=0.01$; $I^{2}=51 \% ; P$ for heterogeneity is 0.08$)$. Exclusion of the other trials did not materially alter results or heterogeneity.

\subsection{Effect of Tai Chi on LDL-C}

LDL-C levels were reported in 4 trials with 288 participants (Tsai et al., 2003; Thomas et al., 2005; Zhang and $\mathrm{Fu}, 2008$; Lu and Kuo, 2012). In comparison with the control group, LDL-C difference between follow-up and baseline in the Tai Chi group demonstrated no significant change (REM: WMD $-1.61 \mathrm{mg} / \mathrm{dl} ; 95 \% \mathrm{CI}-16.25$ to $13.02 \mathrm{mg} / \mathrm{dl} ; P=0.83)$. The forest plot showed significant inter-study heterogeneity overall $\left(I^{2}=92 \%, P<0.00001\right)$ (Fig. 3). Results or heterogeneity did not materially change after exclusion of individual studies respectively during the sensitivity test.

\subsection{Effect of Tai Chi on HDL-C}

Five RCT trials with 392 participants provided data on blood HDL-C (Tsai et al., 2003; Thomas et al., 2005; Zhang and Fu, 2008; Chen et al., 2010; Lu and Kuo, 2012). No significant change was found in HDL-C difference between follow-up and baseline in the Tai Chi group, when compared with the control group (FEM: WMD $0.46 \mathrm{mg} / \mathrm{dl}, 95 \% \mathrm{CI}-0.71$ to $1.64 \mathrm{mg} / \mathrm{dl} ; P=0.44)$. No significant heterogeneity was detected $\left(I^{2}=39 \%, P=0.16\right)$ (Fig. 4). Exclusion of individual studies did not materially alter results. Exclusion of the trial conducted by Lu and Kuo (2012) or Zhang and $\mathrm{Fu}$ (2008) increased heterogeneity across the studies with the same level $\left(I^{2}=54 \%\right.$, $P=0.09$ ).

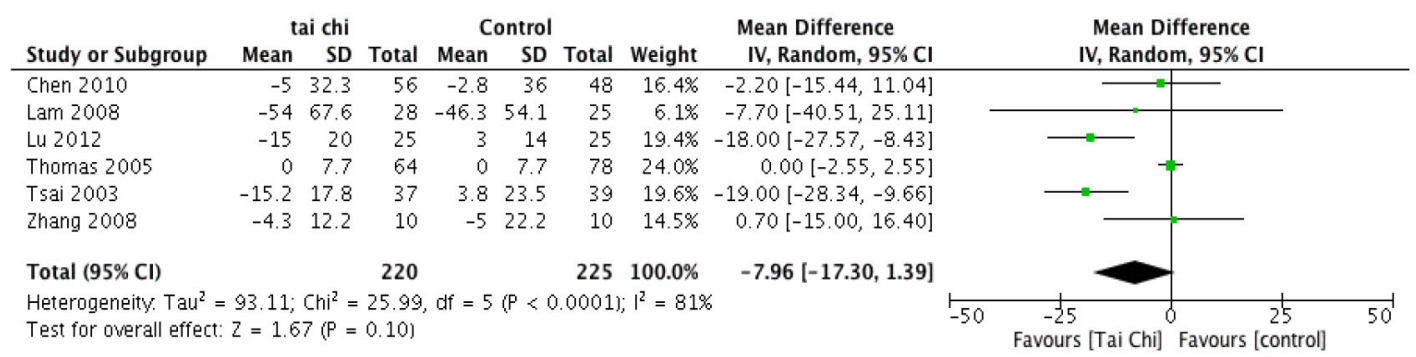

Fig. 2 Forest plot for the effect of Tai Chi on total cholesterol

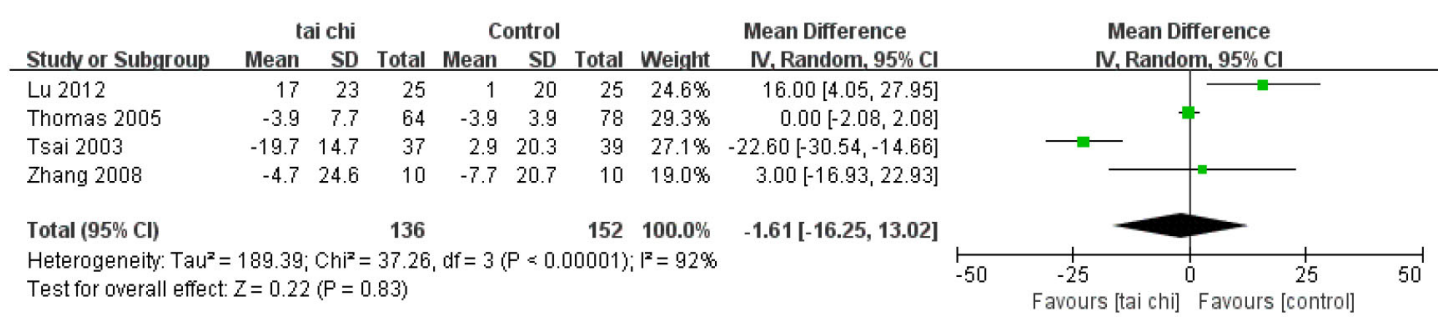

Fig. 3 Forest plot for the effect of Tai Chi on low-density lipoprotein

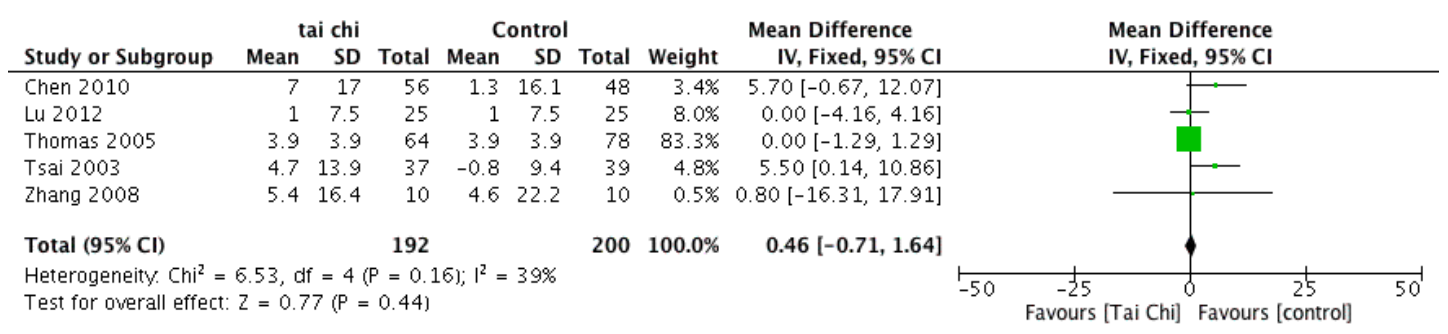

Fig. 4 Forest plot for the effect of Tai Chi on high-density lipoprotein 


\subsection{Effect of Tai Chi on TG}

All 6 studies reported TG level at baseline as well as post-intervention follow-up (Tsai et al., 2003; Thomas et al., 2005; Lam et al., 2008; Zhang and Fu, 2008; Chen et al., 2010; Lu and Kuo, 2012). The aggregated results of these trials suggested that Tai Chi was associated with a significantly decreased blood lipid TG level difference between follow-up and baseline (REM: WMD $-16.81 \mathrm{mg} / \mathrm{dl} ; 95 \% \mathrm{CI}$ -31.27 to $-2.35 \mathrm{mg} / \mathrm{dl} ; P=0.02)$. A significant heterogeneity was detected $\left(I^{2}=70 \%, P=0.005\right)$ (Fig. 5). Notable heterogeneity remained when individual trials were excluded except for the trial conducted by Thomas et al. (2005) $\left(I^{2}=0 \%\right.$; $P$ for heterogeneity is 0.89$)$.

\section{Discussion}

This meta-analysis systematically pooled RCTs with PEDro score more than 3 points to clarify the effect of Tai Chi on lipid profiles. It revealed that practicing Tai Chi could significantly reduce TG. A trend to benefit TC reduction was also observed. As far as we know, the present meta-analysis is the first to investigate the effect of Tai Chi on lipid profiles from RCTs of higher quality.

Tai Chi has been used to improve cardiovascular health for centuries. Scientific evaluation of Tai Chi on lipid profiles has been going on for decades. Channer et al. (1996) revealed that Tai Chi was related to better long-term blood pressure control than aerobic exercise in post-myocardial infarction patients. It was postulated that Tai Chi played a primary or complementary role in primary and secondary prevention in cardiovascular disease, and that improving lipid profiles could be one of the mechanisms (La Forge, 1997). Tsai et al. (2003) conducted the first RCT of Tai Chi on blood lipids in patients with high normal blood pressure and stage I hypertension patients. Twelve weeks of Tai Chi training significantly decreased the serum TC, LDL-C and TG levels, and increased HDL-C by $4.7 \mathrm{mg} / \mathrm{dl}$ from the baseline. No significant change was found in the sedentary life style control group. The beneficial effects of Tai Chi on lipid profiles were confirmed by the trial conducted by Zhang and $\mathrm{Fu}$ (2008). In the trial conducted by Chen et al. (2010), Tai Chi practicing significantly decreased TG and increased HDL-C with no significant effect on TC in comparison with the baseline. Thomas et al. (2005) compared the difference between follow-up and baseline between Tai Chi intervention and a control group, and no significant effects of Tai Chi on lipid profiles were observed. Comparable results were found in the trial of Lam et al. (2008). The limited number of participants in the studies could explain the differing results. Studies with low PEDdro score (Si, 2006; Sha, 2007; Zhou, 2007; Gong et al., 2009; Zhang and Zhang, 2011; Meng, 2014; Shi et al., 2014) were excluded, mostly due to no description on ethical approval, randomization or concealment. Interestingly most studies with lower quality revealed that Tai Chi significantly decreased TG, TG, and LDL-C, and increased HDL-C in varied populations (Si, 2006; Sha, 2007; Zhou, 2007; Gong et al., 2009; Zhang and Zhang, 2011; Meng, 2014; Shi et al., 2014). With a thorough synthesis from qualified RCT, we found that Tai Chi decreased TG, showed a trend to decrease TC, and had no significant effect on LDL-C or HDL-C in the given time. Interestingly, a newly published metaanalysis (Cai and Zou, 2015) synthesized RCTs of aerobic exercise, mostly lasing for 12 and 24 weeks, on blood lipid profiles in obese or overweight adults. The analysis showed that only TG level was significantly decreased, which is comparable to our findings.

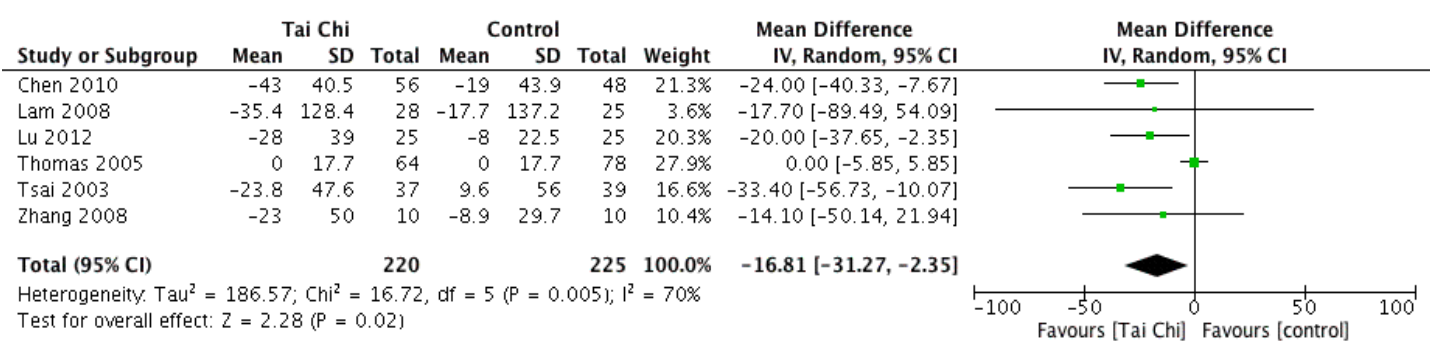

Fig. 5 Forest plot for the effect of Tai Chi on triglycerides 
The trials included in our meta-analysis were of relatively short duration, mostly within three months (Tsai et al., 2003; Zhang and Fu, 2008; Chen et al., 2010; Lu and Kuo, 2012). The results coincided with the fact that in comparison with TG, LDL-C and HDL-C needed a longer time to change by the means of exercise and complementary intervention (Franklin et al., 2014; Gordon et al., 2014; Noland, 2015). The baseline LDL-C and HDL-C levels were nearly normal, which increased the difficulty in evaluating the treatment effect of Tai Chi on dyslipidemias. Furthermore, substracting the baseline value from the follow-up led to smaller numerical scale and possibly larger SD, which statistically required more participants and longer duration.

Tai Chi improved the lipid profiles resulting from the beneficial effects of inherent exercise enhanced by traditional Chinese qi lead and meditation. Beebe et al. (2013) revealed that Tai Chi exercise was associated with improvements in LDL-C particle size in obese older women. Tai Chi practicing comprises aerobic exercise and resistance exercise, increasing the whole body fat oxidation, adipose tissue lipolysis and fatty acid utilization by skeletal muscle, mobilizing lipid from adipose tissue, liver and intramuscular reserves, dictating tissue fatty acid uptake, intracellular lipid delivery and mitochondrial $\beta$-oxidation, coordinating activation of the sympathetic nervous system and induction of energy deficit-sensing pathways, promoting energy expenditure via heightened TG/FA cycling, inducing energy-sensing pathways such as adenosine 5'-monophosphate (AMP)activated protein kinase (AMPK), activating peroxisome proliferator-activated receptor delta (PPARD)mediated remodeling of lipid metabolism pathways in muscle, and increasing adiponectin level with strong anti-atherogenic and anti-inflammatory effects (Chang et al., 2011; Franklin et al., 2014; Gordon et al., 2014; Noland, 2015). The possibility that the change of body fat ratio and insulin resistance might have an influence on lipid profiles should also be considered (Kohno et al., 2000).

Limitations of this meta-analysis should be acknowledged. Firstly, the included RCTs generally had a modest number participants and limited follow-up. Secondly, the meta-analysis did not report treatment effect systematically, such as by risk stratification. The included participants were of low to medium cardiovascular risk. The baseline lipid profile characteristics were close to normal. Thirdly, some participants were on lipid-lowering medication, though they were asked to maintain the identical agent and dosage during the follow-up. Fourthly, the diversity of Tai Chi exercise prescription in the included studies, including the type, exercise duration, and the frequency, limits the ability to make firm conclusions. Fifthly, participants in the Tai Chi groups might have higher expectations of the treatment results since double blind was practically unavailable, and bias of assignments' awareness was inevitably introduced. The limitations, together with obesity, diet and baseline lipid profile characteristics, remained the origin of heterogeneity across the trials. Focusing on these aspects may be of interest for future research on the subject.

Despite its limitations, our meta-analysis still remains clinically valuable. The findings suggest that Tai Chi decreases TG and probably TC, and could be a valuable addition to a lipid-lowering program. The exercise intensity is low to moderate, which is eligible and feasible for the elderly. Tai Chi has multidimensional effects, which benefits the individuals with multiple risk factors and diseases. It remains promising to integrate Tai Chi into current and future health care to treat dyslipidemias.

\section{Conclusions}

Our meta-analysis is the first to comprehensively examine the effect of Tai Chi on lipid profiles with RCTs. It suggests that Tai Chi practicing is associated with improvement of blood lipid profiles. Tai Chi exercise significantly lowers TG level and shows a tendency to decrease TC level. Overall, there remains a need for larger, longer, higher quality trials to address the relationship between a Tai Chi exercise regimen (intensity, amount, duration) and lipid profile change.

\section{Compliance with ethics guidelines}

Xiao-hong PAN, Amina MAHEMUTI, Xue-hua ZHANG, Ya-ping WANG, Po HU, Ju-bo JIANG, Mei-xiang XIANG, Gang LIU, and Jian-an WANG declare that they have no conflict of interest.

This article does not contain any studies with human or animal subjects performed by any of the authors. 


\section{References}

Beebe, N., Magnanti, S., Katkowski, L., et al., 2013. Effects of the addition of $t$ 'ai chi to a dietary weight loss program on lipoprotein atherogenicity in obese older women. $J$. Altern. Complement. Med., 19(9):759-766. http://dx.doi.org/10.1089/acm.2012.0531

Cai, M., Zou, Z., 2015. Effect of aerobic exercise on blood lipid and glucose in obese or overweight adults: a metaanalysis of randomised controlled trials. Obes. Res. Clin. Pract., in press. http://dx.doi.org/10.1016/j.orcp.2015.10.010

Chang, R.Y., Koo, M., Ho, M.Y., et al., 2011. Effects of Tai Chi on adiponectin and glucose homeostasis in individuals with cardiovascular risk factors. Eur. J. Appl. Physiol., 111(1):57-66 http://dx.doi.org/10.1007/s00421-010-1628-y

Channer, K.S., Barrow, D., Barrow, R., et al., 1996. Changes in haemodynamic parameters following Tai Chi Chuan and aerobic exercise in patients recovering from acute myocardial infarction. Postgrad. Med. J., 72(848):349-351.

Chen, S.C., Ueng, K.C., Lee, S.H., et al., 2010. Effect of t'ai chi exercise on biochemical profiles and oxidative stress indicators in obese patients with type 2 diabetes. J. Altern. Complement. Med., 16(11):1153-1159. http://dx.doi.org/10.1089/acm.2009.0560

Dalusung-Angosta, A., 2011. The impact of Tai Chi exercise on coronary heart disease: a systematic review. J. Am. Acad. Nurse Pract., 23(7):376-381. http://dx.doi.org/10.1111/j.1745-7599.2011.00597.x

Franklin, B.A., Durstine, J.L., Roberts, C.K., et al., 2014. Impact of diet and exercise on lipid management in the modern era. Best Pract. Res. Clin. Endocrinol. Metab., 28(3):405-421. http://dx.doi.org/10.1016/j.beem.2014.01.005

Frishman, W.H., Beravol, P., Carosella, C., 2009. Alternative and complementary medicine for preventing and treating cardiovascular disease. Dis. Mon., 55(3):121-192. http://dx.doi.org/10.1016/j.disamonth.2008.12.002

Gong, J.C., Liu, Y.D., Zhao, D.M., et al., 2009. Taijishan effect on community indicators of blood lipid in middle aged and old women. J. Commun. Med., 7(17):34-35 (in Chinese).

Gordon, B., Chen, S., Durstine, J.L., 2014. The effects of exercise training on the traditional lipid profile and beyond. Curr. Sports Med. Rep., 13(4):253-259. http://dx.doi.org/10.1249/JSR.0000000000000073

Higgins, J.P., Deeks, J., 2011. Obtaining standard deviations from standard errors and confidence intervals for group means. In: Higgins, J.P., Green, S. (Eds.), Cochrane Handbook for Systematic Reviews of Interventions. Version 5, the Cochrane Collaboration, 7.7.3.3.

Higgins, J.P., Thompson, S.G., Deeks, J.J., et al., 2003. Measuring inconsistency in meta-analyses. $B M J, 327(7414)$ : 557-560.

http://dx.doi.org/10.1136/bmj.327.7414.557

Hu, P., Huang, M.Y., Hu, X.Y., et al., 2015. Meta-analysis of
C242T polymorphism in CYBA genes: risk of acute coronary syndrome is lower in Asians but not in Caucasians. J. Zhejiang Univ.-Sci. B (Biomed. \& Biotechnol.), 16(5): 370-379.

http://dx.doi.org/10.1631/jzus.B1400241

Hui, S.S., Xie, Y.J., Woo, J., et al., 2015. Effects of Tai Chi and walking exercises on weight loss, metabolic syndrome parameters, and bone mineral density: a cluster randomized controlled trial. Evid. Based Complement. Altern. Med., 2015:976123. http://dx.doi.org/10.1155/2015/976123

Kohno, K., Matsuoka, H., Takenaka, K., et al., 2000. Depressor effect by exercise training is associated with amelioration of hyperinsulinemia and sympathetic overactivity. Intern. Med., 39(12):1013-1019.

La Forge, R., 1997. Mind-body fitness: encouraging prospects for primary and secondary prevention. J. Cardiovasc. Nurs., 11(3):53-65. http://dx.doi.org/10.1097/00005082-199704000-00006

Lam, P., Dennis, S.M., Diamond, T.H., et al., 2008. Improving glycaemic and BP control in type 2 diabetes. The effectiveness of tai chi. Aust. Fam. Physician, 37(10):884-887.

Lu, W.A., Kuo, C.D., 2012. Effect of 3-month Tai Chi Chuan on heart rate variability, blood lipid and cytokine profiles in middle-aged and elderly individuals. Int. J. Gerontol., 6(4):267-272.

http://dx.doi.org/10.1016/j.ijge.2012.01.025

Maher, C.G., Sherrington, C., Herbert, R.D., et al., 2003. Reliability of the PEDro scale for rating quality of randomized controlled trials. Phys. Ther., 83(8):713-721.

Meng, E., 2014. The effect of Taijiquan on lipid profile and insulin resistance in type II diabetes. Chin. J. Gerontol., 34(19):5358-5360 (in Chinese).

Moher, D., Liberati, A., Tetzlaff, J., et al., 2009. Preferred reporting items for systematic reviews and meta-analyses: the PRISMA Statement. $B M J, 339: \mathrm{b} 2535$.

http://dx.doi.org/10.1136/bmj.b2535

Mozaffarian, D., Benjamin, E.J., Go, A.S., et al., 2015. Heart disease and stroke statistics-2015 update: a report from the American Heart Association. Circulation, 131(4): e29-e322. http://dx.doi.org/10.1161/CIR.0000000000000152

Nicholls, S.J., Pisaniello, A.D., Kataoka, Y., et al., 2014. Lipid pharmacotherapy for treatment of atherosclerosis. Expert Opin. Pharmacother., 15(8):1119-1125. http://dx.doi.org/10.1517/14656566.2014.904287

Noland, R.C., 2015. Exercise and regulation of lipid metabolism. Prog. Mol. Biol. Transl. Sci., 135:39-74. http://dx.doi.org/10.1016/bs.pmbts.2015.06.017

Pan, L., Yan, J., Guo, Y., et al., 2013. Effects of Tai Chi training on exercise capacity and quality of life in patients with chronic heart failure: a meta-analysis. Eur. J. Heart Fail., 15(3):316-323. http://dx.doi.org/10.1093/eurjhf/hfs 170

Reiner, Z., Catapano, A.L., de Backer, G., et al., 2011. ESC/EAS Guidelines for the management of dyslipidaemias. Eur. Heart J., 32(14):1769-1818. http://dx.doi.org/10.1093/eurheartj/ehr158 
Sha, P., 2007. Effect of Taijiquan exercise on the nitrogen monoxidum and blood lipid of elder patients with atherosclerosis. J. Clin. Rehab. Tissue Eng. Res., 11(34): 6832-6834 (in Chinese).

Shi, Z.M., Wen, H.P., Liu, F.R., et al., 2014. The effects of tai chi on the renal and cardiac functions of patients with chronic kidney and cardiovascular diseases. J. Phys. Ther. Sci., 26(11):1733-1736. http://dx.doi.org/10.1589/jpts.26.1733

Si, C.J., 2006. The effect of Taijiquan exercise on the constitution of mid and old age people. J. Liaoning Normal Univ. (Nat. Sci. Ed.), 29(3):379-381 (in Chinese).

Stone, N.J., Robinson, J.G., Lichtenstein, A.H., et al., 2014. 2013 ACC/AHA guideline on the treatment of blood cholesterol to reduce atherosclerotic cardiovascular risk in adults: a report of the American College of Cardiology/ American Heart Association Task Force on Practice Guidelines. Circulation, 129(25 Suppl. 2):S1-S45. http://dx.doi.org/10.1161/01.cir.0000437738.63853.7a

Thomas, G.N., Hong, A.W., Tomlinson, B., et al., 2005. Effects of Tai Chi and resistance training on cardiovascular risk factors in elderly Chinese subjects: a 12-month longitudinal, randomized, controlled intervention study. Clin. Endocrinol. (Oxf.), 63(6):663-669.

Tsai, J.C., Wang, W.H., Chan, P., et al., 2003. The beneficial effects of Tai Chi Chuan on blood pressure and lipid profile and anxiety status in a randomized controlled trial. J. Altern. Complement. Med., 9(5):747-754.

Verhagen, A.P., de Vet, H.C., de Bie, R.A. et al., 1998. The Delphi list: a criteria list for quality assessment of randomized clinical trials for conducting systematic reviews developed by Delphi consensus. J. Clin. Epidemiol., 51(12):1235-1241. http://dx.doi.org/10.1016/S0895-4356(98)00131-0

Walach, H., Jonas, W.B., 2004. Placebo research: the evidence base for harnessing self-healing capacities. J. Altern. Complement. Med., 10(Suppl. 1):S103-S112.
Wayne, P.M., Kaptchuk, T.J., 2008. Challenges inherent to $t^{\prime} a i$ chi research: Part I-t'ai chi as a complex multicomponent intervention. J. Altern. Complement. Med., 14(1): 95-102.

http://dx.doi.org/10.1089/acm.2007.7170A

Zhang, H.L., Zhang, H.J., 2011. Effects of long-term Taijiquan practice on lipid metabolism and related hormone levels of obese university students. J. Shenyang Sport Univ., 30(6):95-98 (in Chinese).

Zhang, Y., Fu, F.H., 2008. Effects of 14-week Tai Ji Quan exercise on metabolic control in women with type 2 diabetes. Am. J. Chin. Med., 36(4):647-654.

Zhou, S.W., 2007. Effects of Tai Ji Quan on blood pressure and lipid profile in patients with stage I essential hypertension. Int. Med. Health Guid. News, 13(15):60-64 (in Chinese).

\section{中文概要}

题 目: 太极锻炼对血脂的临床疗效: 随机对照试验的 meta 分析

目 的: 探讨太极锻炼是否能改善血脂水平。

创新点: 明确太极锻炼对血脂的影响, 为非药物调脂治疗 提供新手段。

方 法: 通过篮选太极锻炼持续 4 周以上, PEDro 评分 3 分以上, 且观察太极锻炼对血脂影响的成人随机 对照试验, meta 分析太极锻炼对总胆固醇、低密 度脂蛋白胆固醇、高密度脂蛋白胆固醇和甘油三 酯的影响。

结 论: 通过 meta 分析最终入选的 6 项研究 (共 445 例患 者) 的结果显示, 太极锻炼能显著降低血甘油三 酯水平, 且有降低总胆固醇的趋势, 但是对于低 密度脂蛋白胆固醇和高密度脂蛋白胆固醇未发 现有明显影响。

关键词: 太极; 血脂; 甘油三酯; Meta 分析 